\title{
Effectiveness of mirror therapy on upper limb function, activities of daily living, and depression in post-stroke depression patients
}

\author{
Xiang Zhang $®$, Yi Zhang $®$, Yu Liu@ $®$, Qiujin Yao $®$ \\ Department of Rehabilitation, The Third Affiliated Hospital of Soochow University, Changzhou, China
}

Received: May 18, 2020 Accepted: August 31, 2020 Published online: September 01, 2021

\begin{abstract}
Objectives: This study aims to investigate the effects of mirror therapy (MT) on upper limb function, activities of daily living (ADLs), and depression in post-stroke depression patients.

Patients and methods: Between November 2018 and December 2019, a total of 60 post-stroke patients (33 males, 27 females; mean age: $58.45 \pm 11.13$ years; range, 35 to 88 years) were included. The patients were randomly divided into either the cosntrol group ( $\mathrm{n}=30$ ) or the MT group ( $n=30$ ). Regular occupational therapy was provided for the control group (two times per day for 30 min per session, five times per week over four weeks). Occupational therapy and MT were used to treat patients in the mirror group (one 30 min session once per day, five times per week over four weeks). Motor function (Fugl-Meyer Assessment of the Upper Extremity, FMA-UE), ADL (Modified Barthel Index, MBI) and depression (17-item Hamilton Depression Scale, HAMD-17) were used to evaluate the treatment outcomes.

Results: Before treatment, the mean HAMD-17, FMA-UE, and MBI scores showed no significant difference between the two groups ( $p>0.05)$. After treatment, the mirror group exhibited more significant improvements than the control group in terms of the mean HAMD-17, FM-UE, and MBI ( $<<0.05)$. After four weeks, the mean FMA-UE and MBI scores revealed more significant improvements than the baseline scores in the control group $(\mathrm{p}<0.01)$. The mean HAMD-17, FMA-UE, and MBI scores showed more significant improvements than the baseline scores in the MT group $(\mathrm{p}<0.001)$.
\end{abstract}

Conclusion: Based on these results, MT can effectively improve motor function, ADLs, and depression in post-stroke depression patients. The curative effectiveness of MT seems to be more prominent than the regular occupational therapy.

Keywords: Activities of daily living, depression, mirror therapy, post-stroke depression, upper limb function.

Stroke ranks the second most common cause of death in the world and is one of the major causes of disability. ${ }^{[1]}$ The worldwide morbidity rate and prevalence rate of stroke and its syndromes have been increasing annually. ${ }^{[2]}$ In 2017, across the world, stroke contributed to 6.17 million deaths. ${ }^{[3]}$ In addition, survivors experience different function impairments, while $33 \%$ of survivors develop post-stroke depression (PSD). ${ }^{[4]}$ A research on ischemic stroke patients demonstrated that PSD patients, compared to those having no PSD, had a 15\% higher disability rate, were hospitalized for a longer period, and their rehabilitation treatment was less effective than that of non-PSD patients. ${ }^{[5]}$ Also, PSD has a negative impact on patients' daily living activities (ADLs) and quality of life, and it increases the mortality and suicide rate, thereby, placing a heavy burden on families and the society. ${ }^{[6]}$

Mirror therapy (MT), also known as mirror visual feedback, was first developed in 1995 by Ramachandran et al., ${ }^{[7]}$ to treat phantom limb pain. Altschuler ${ }^{[8]}$ was the first to apply MT to aid recovery of upper limb function in PSD patients. In a meta-analysis carried out by Thieme et al., ${ }^{[9]}$ MT could significantly improve the upper and lower limb motor functions and other functions that support ADLs. The mechanism of

Corresponding author: Zhang Yi, MD. Department of Rehabilitation, The Third Affiliated Hospital of Soochow University, 213000 Changzhou, China. 
MT involves activating relevant brain regions, such as the frontal lobe. ${ }^{[10]}$ Castanheira et al. ${ }^{[1]}$ found that the frontal lobe and anterior cingulate gyrus were the main brain areas in which function was affected in patients with depression.

There is a limited number of data in the literature on this subject. In the present study, we hypothesized that MT could exert a positive influence on improving PSD. We, therefore, aimed to examine the effect of MT on the treatment of depressive emotions of PSD patients and on the recovery of PSD patients' upper limb function and their ADLs.

\section{PATIENTS AND METHODS}

This randomized controlled trial study was conducted at The Third Affiliated Hospital of Soochow University, Department of Rehabilitation between November 2018 and December 2019. A total of 60 post-stroke patients (33 males, 27 females; mean age: $58.45 \pm 11.13$ years; range, 35 to 88 years) who were hospitalized in our center were included in the study. Inclusion criteria were as follows: patients who were first-time stroke patients with a confirmed diagnosis by computed tomography and magnetic resonance imaging; patients who met the Diagnostic and Statistical Manual of Mental Disorders-V (DSM-V) criteria for depressive disorder; patients who suffered from hemiplegia with upper limb functions (Brunnstrom Stage 1-4; setting balance level $>$ I, limb muscle tension improvement level $\leq 2$, as assessed by the he Modified Ashworth Scale [MAS]); patients who were in the lucid state of mind and able to understand simple instructions and to cooperate with the treatment; patients with a 17-item Hamilton Depression Scale (HAMD-17) of $\geq 7$; patients having normal vision without no visual field defect; and patients and/or their relatives who were aware of and willing to give a consent for the study. Exclusion criteria were as follows: having upper limb bone fractures or other injuries and having a history of depression before the stroke; using medical drugs which may affect the mood; and previous MT. Using randomized numbers, we allocated the patients into two groups as follows: the control group $(n=30)$ and the MT group $(n=30)$. A written informed consent was obtained from each patient. The study protocol was approved by the Ethics Committee of The Third Affiliated Hospital of Soochow University (No: 2018032). The study was conducted in accordance with the principles of the Declaration of Helsinki.

\section{Intervention methods}

The control group received occupational therapy for five days per week, two sessions per day, 30 min per session over a total of four weeks. Occupational therapy consisted of therapeutic occupational activity training, sensory training, and ADL training. The content of the training was developed by the therapists for each individual patient. The baseline treatment lasted for four weeks, the frequency was one session five days per week, and the duration of each session was $1 \mathrm{~h}$.

The MT group received occupational therapy for one session per day for five days per week, $30 \mathrm{~min}$ per session over a total of four weeks. Based on the occupational therapy, MT was also provided throughout the whole course (one session per day for five days per week and each session lasted $1 \mathrm{~h}$ ).

During the MT, the patients were instructed to sit up straight and put their two upper limbs on the table. A mirror was placed on the midsagittal plane between the limbs. The front of the mirror faced toward the healthy part, while its back faced toward the dysfunctional part. The patients were instructed to lean their body slightly toward the healthy part so that they could observe the mirror image of their healthy upper limb's movement, while their dysfunctional part, hidden by the mirror, remained unseen. Quietness was maintained in the therapeutic environment. Based on the degree of difficulty, the researchers instructed the patients to make 10 movements using their healthy upper limb in the following order: flexion and tension of the elbow joint, palmar flexion and dorsal extension of the wrist, wrist ulnar deviation and radial deviation, making a thumb-up gesture, an empty-hand grip, hook-form grip, cylindrical form grip, sphere form grip, finger-to-finger movement, and side pinch. The patients were instructed to observe the mirror image.

\section{Assessment}

Before the treatment and at the end of four-week treatment, the motor functions, ADL function, and depression level were evaluated by two medical staff members from the recovery department who had a psychological consultation certificate and did not participate in the research.

The motor functions were evaluated using the Fugl-Meyer Upper Extremity (FMA-UE) Scale which is a widely used and highly recommended scale to assess upper limb function in post-stroke patients. ${ }^{[12]}$ It consists of 33 items, each of which is assigned a score ranging from 0 to 2 . The total score is 66 . A higher score indicates higher performance. 
The performance in ADLs was evaluated using the Modified Barthel Index (MBI). It consists of 10 items, with a total score of 100 . Higher scores indicate a higher degree of performance. This scale is easy to use and has high reliability and validity. ${ }^{[13]}$ It is, therefore, the most widely used method to evaluate ADL capability.

Severity of depression was evaluated using the HAMD-17 scale. It is the most widely used scale to evaluate depression worldwide. The HAMD-17 scale consists of 17 items, and each is assigned a score ranging from between 1 and 4 . Higher score indicates a greater severity of depression. The highest total score is 68 (0-7 without depression, 8-17 mild depression, 18-24 moderate depression, higher than 24 severe depression). ${ }^{[14,15]}$

\section{Statistical analysis}

Statistical analysis was performed using the IBM SPSS for Windows version 21.0 software (IBM Corp., Armonk, NY, USA). Descriptive data were expressed in mean \pm standard deviation (SD), median (min$\max )$ or number and frequency, where applicable. The normality of data distribution was assessed using the Kolmogorov-Smirnov test. For the intra-group analysis and pre- and post-treatment comparison, a paired samples t-test and Wilcoxon signed-rank test were carried out for normally and non-normally distributed data. For the inter-group analysis, an independent samples t-test was conducted for normally distributed data and the Mann-Whitney $U$ test was employed for non-normally distributed data. A $p$ value of $<0.05$ was considered statistically significant.

\section{RESULTS}

Baseline demographic characteristics of the study population are shown in Table 1.

The mean FMA-UE and MBI scores of the control group showed a significant improvement after the treatment $(\mathrm{p}<0.01)$. The mean HAMD-17, FMA-UE, and MBI scores of the MT group also significantly improved after the treatment $(\mathrm{p}<0.01)$ (Table 2).

Between the groups, there was no significant difference in the mean HAMD-17, FMA-UE, and MBI scores at baseline ( $p>0.05)$. However, after the treatment, the mean HAMD-17 scores were found to be significantly lower in the MT group $(\mathrm{p}<0.05)$. Furthermore, the mirror group showed a significant improvement in the mean FMA-UE and MBI scores after the treatment $(\mathrm{p}<0.001)$ (Table 2).

TABLE 1

Baseline demographic characteristics of study population

\begin{tabular}{|c|c|c|c|c|c|}
\hline & \multirow[b]{2}{*}{ Sample number } & \multicolumn{2}{|c|}{ Sex } & \multirow{2}{*}{$\begin{array}{l}\text { Age (year) } \\
\text { Mean } \pm S D\end{array}$} & \multirow{2}{*}{$\frac{\text { Disease duration (day) }}{\text { Mean } \pm \mathrm{SD}}$} \\
\hline & & Male & Female & & \\
\hline Control group & 30 & 18 & 12 & $59.9 \pm 11.8$ & $31.5 \pm 8.9$ \\
\hline Mirror group & 30 & 15 & 15 & $57.0 \pm 10.4$ & $29.5 \pm 7.8$ \\
\hline Statistics value & & \multicolumn{2}{|c|}{$\chi^{2}=0.606$} & $\mathrm{t}=1.033$ & $\mathrm{t}=0.942$ \\
\hline $\mathrm{P}$-value & & \multicolumn{2}{|c|}{0.436} & 0.306 & 0.350 \\
\hline
\end{tabular}

\begin{tabular}{|c|c|c|c|c|}
\hline \multicolumn{5}{|c|}{$\begin{array}{c}\text { TABLE } 2 \\
\text { Comparison of subject performance of study group }\end{array}$} \\
\hline \multirow[b]{2}{*}{ Group } & \multirow[b]{2}{*}{ Sample number } & \multirow{2}{*}{$\begin{array}{l}\text { HAMD-17 } \\
\text { Mean } \pm \text { SD }\end{array}$} & \multirow{2}{*}{$\begin{array}{c}\text { FM-UE } \\
\text { Mean } \pm \text { SD }\end{array}$} & \multirow{2}{*}{$\frac{\text { MBI }}{\text { Mean } \pm \text { SD }}$} \\
\hline & & & & \\
\hline \multicolumn{5}{|c|}{ Control group } \\
\hline Before & 30 & $12.2 \pm 3.9$ & $15.6 \pm 3.9$ & $35.9 \pm 8.8$ \\
\hline After & 30 & $12.6 \pm 4.4$ & $20.0 \pm 5.1^{\mathrm{b}}$ & $43.1 \pm 10.0^{\mathrm{b}}$ \\
\hline \multicolumn{5}{|c|}{ Mirror group } \\
\hline Before & 30 & $12.0 \pm 3.4$ & $16.8 \pm 5.0$ & $38.7 \pm 10.0$ \\
\hline After & 30 & $10.3 \pm 4.0^{\mathrm{ac}}$ & $26.3 \pm 6.4^{\mathrm{bd}}$ & $58.0 \pm 10.3^{\mathrm{bd}}$ \\
\hline
\end{tabular}




\section{DISCUSSION}

In the present study, we examined the effect of MT on the treatment of depressive emotions of PSD patients and on the recovery of PSD patients' upper limb function and their ADLs to pave the way for further studies developing specific effective treatments for PSD patients. Our study results showed that MT could effectively improve motor function, ADLs, and depression in PSD patients.

Post-stroke depression is a psychological disorder which is frequently observed after stroke. Studies have shown that the morbidity rate of PSD reaches 33\%, with females (5.9 to $78.3 \%$ ) being more vulnerable than males (4.7 to $66.7 \%)^{[16,17]}$ The cause of PSD has not been fully understood, yet. It is believed that several factors including neurological factors, such as neural function defects and socio-psychological factors (e.g., social support and family relations) play a role in its etiology.

Currently, PSD treatments include antidepressants, psychological therapy, and transcranial magnetic stimulation (TMS). ${ }^{[4]}$ However, researches have shown that there is no evidence supporting the PSD treatment. ${ }^{[18]}$ Paroxetine may be an optimal choice for PSD medical treatment, although it has low physiological tolerance and may cause negative side effects. ${ }^{[19]}$ Cognitive-behavioral therapies also help to improve depressive symptoms, whereas such improvements have yet to be verified by well-designed, randomized-controlled trials. ${ }^{[20]}$ Repetitive TMS can be a beneficial treatment; however, further studies are needed to establish the stimulation parameters and locations. ${ }^{[21]}$ In our study, the use of MT to treat PSD showed that the mirror group had significantly lower HAMD-17 scores after the four-week treatment compared to baseline. This finding indicates that MT can help to mitigate depression in PSD patients. This is possibly due to MT-induced activation of the cortex and other brain regions that are relevant to the patients' depressive emotions. ${ }^{[10,11]}$ Following activation of the cortex, MT may exert a positive influence on the patients' emotions.

Apart from the discussion on the influence of MT on PSD patients' depression, we also observed MT's influence on PSD patients' upper limb function and ADL. The FMA-UE scale was used to evaluate the patients' upper limb function. Concerning the FMA-UE performance, the mirror group showed a significant improvement after the four-week treatment, and the improvement was more significant than that observed in the control group. These findings indicate shows that MT is also effective in improving PSD patients' upper limb function and the effect is more prominent than conventional treatment, consistent with previous studies. In a Cochrane review including 36 studies, MT was found to more significantly improve motor function in post-stroke patients, compared to other treatment modalities. ${ }^{[9]}$ Although the mechanism of how MT improves motor function has not been completely understood yet, it has been proposed that MT prompts the healthy brain hemisphere to interact with the disordered brain hemisphere, thereby, activating related brain regions and promoting the reconstruction of the cortex neural network, which helps to modify cortex excitability and promote functional recovery.

Activities of daily living are the most basic and universal activities which humans must repeat every day to survive and adapt to the environment. The ADL scales are used to evaluate patients' disabilities, their quality of life, and functional conditions. ${ }^{[22]}$ The MBI is one of the most commonly used and researched ADL assessment scales. In our study, the mirror group had significantly higher MBI performance after the four-week treatment, compared to pre-treatment performance. Of note, MT was used to train the fine motor skills of the patients' elbows, wrists, shoulders, and fingers in this study. These skills are important for ADLs, such as eating, dressing, etc. While improving the upper limb function, MT can also improve the PSD patients' ADL skills.

The relatively low sample size is the main limitation of the present study. Further large-scale, multi-center, randomized controlled studies are needed to confirm these findings. In addition, patients having mild depression only were included in this study and further researches are warranted to evaluate whether MT can be helpful in case of moderate or severe depression. The lack of a long-term follow-up is another limitation, which requires further investigation to gain a better understanding of the underlying mechanism of MT in PSD treatment.

In conclusion, this is the first study to utilize MT in PSD patients and discuss its influence on the patients' motor functions, ADLs, and depression. Based on these results, MT can improve motor function, ADL, and depression in PSD patients and its effect is more prominent than that of conventional treatment.

\section{Declaration of conflicting interests}

The authors declared no conflicts of interest with respect to the authorship and/or publication of this article. 


\section{Funding}

The authors received no financial support for the research and/or authorship of this article.

\section{REFERENCES}

1. Hankey GJ. Stroke. Lancet 2017;389:641-54.

2. Feigin VL, Forouzanfar MH, Krishnamurthi R, Mensah GA, Connor M, Bennett DA, et al. Global and regional burden of stroke during 1990-2010: findings from the Global Burden of Disease Study 2010. Lancet 2014;383:245-54.

3. Dong S, Fang J, Li Y, Ma M, Hong Y, He L. The population attributable risk and clustering of stroke risk factors in different economical regions of China. Medicine (Baltimore) 2020;99:e19689.

4. Starkstein SE, Hayhow BD. Treatment of Post-Stroke Depression. Curr Treat Options Neurol 2019;21:31.

5. Paolucci S, Iosa M, Coiro P, Venturiero V, Savo A, De Angelis D, et al. Post-stroke depression increases disability more than $15 \%$ in ischemic stroke survivors: A case-control study. Front Neurol 2019;10:926.

6. Mitchell AJ, Sheth B, Gill J, Yadegarfar M, Stubbs B, Yadegarfar M, et al. Prevalence and predictors of post-stroke mood disorders: A meta-analysis and meta-regression of depression, anxiety and adjustment disorder. Gen Hosp Psychiatry 2017;47:48-60.

7. Ramachandran VS, Rogers-Ramachandran D, Cobb S. Touching the phantom limb. Nature 1995;377:489-90.

8. Altschuler EL. Motor rehabilitation in a stroke patient using a mirror. 28th Annual Meeting, Los Angeles, Calif., November 7-12, 1998. Society for Neuroscience Abstracts; 1998;24:1408.

9. Thieme H, Morkisch N, Mehrholz J, Pohl M, Behrens J, Borgetto B, et al. Mirror therapy for improving motor function after stroke. Cochrane Database Syst Rev 2018;50:CD008449.

10. Milde C, Rance M, Kirsch P, Trojan J, Fuchs X, Foell J, et al. Do mirror glasses have the same effect on brain activity as a mirror box? Evidence from a functional magnetic resonance imaging study with healthy subjects. PLoS One 2015;10:e0127694.

11. Castanheira L, Silva C, Cheniaux E, Telles-Correia D. Neuroimaging Correlates of Depression-Implications to Clinical Practice. Front Psychiatry 2019;10:703.

12. Singer B, Garcia-Vega J. The fugl-meyer upper extremity scale. J Physiother 2017;63:53.

13. Leung SO, Chan CC, Shah S. Development of a Chinese version of the Modified Barthel Index-- validity and reliability. Clin Rehabil 2007;21:912-22.

14. Hamilton M. Development of a rating scale for primary depressive illness. Br J Soc Clin Psychol 1967;6:278-96.

15. Zheng YP, Zhao JP, Phillips M, Liu JB, Cai MF, Sun SQ, et al. Validity and reliability of the Chinese Hamilton Depression Rating Scale. Br J Psychiatry 1988;152:660-4.

16. Das J, G K R. Post stroke depression: The sequelae of cerebral stroke. Neurosci Biobehav Rev 2018;90:104-14.

17. Ayis SA, Rudd AG, Ayerbe L, Wolfe CDA. Sex differences in trajectories of depression symptoms and associations with 10-year mortality in patients with stroke: the South London Stroke Register. Eur J Neurol 2019;26:872-9.

18. Hackett ML, Anderson CS, House A, Xia J. Interventions for treating depression after stroke. Cochrane Database Syst Rev 2008;(4):CD003437.

19. Deng L, Qiu S, Yang Y, Wang L, Li Y, Lin J, et al. Efficacy and tolerability of pharmacotherapy for post-stroke depression: a network meta-analysis. Oncotarget 2018;9:23718-28.

20. Wang SB, Wang YY, Zhang QE, Wu SL, Ng CH, Ungvari GS, et al. Cognitive behavioral therapy for post-stroke depression: A meta-analysis. J Affect Disord 2018;235:589-96.

21. Shen X, Liu M, Cheng Y, Jia C, Pan X, Gou Q, et al. Repetitive transcranial magnetic stimulation for the treatment of poststroke depression: A systematic review and meta-analysis of randomized controlled clinical trials. J Affect Disord 2017;211:65-74.

22. Wiener JM, Hanley RJ, Clark R, Van Nostrand JF. Measuring the activities of daily living: comparisons across national surveys. J Gerontol 1990;45:S229-37. 